\title{
Poisson noise reduction in deconvolution microscopy
}

\author{
Murillo R.P. Homem ${ }^{1}$, Marcelo R. Zorzan² and Nelson D.A. Mascarenhas ${ }^{3}$
}

Manuscript received on December 21, 2011 / accepted on December 30, 2011

\begin{abstract}
Computational optical sectioning microscopy is a powerful tool to reconstruct three-dimensional images from optical two-dimensional sections of a biological specimen acquired by means of a fluorescence microscope. Due to limiting factors in the imaging systems, the images are degraded by both the optical system and detection process. Each of the two-dimensional section of the three-dimensional data set are blurred by contributions of light from other out-of-focus planes. Besides, they are also corrupted by noise due to quantum fluctuations of light. In this work we present a method to perform the restoration of three-dimensional data obtained by fluorescence microscopy. The algorithm consists of the use of a noise reduction procedure based on the Anscombe transformation followed by the Richardson-Lucy deconvolution algorithm. Results showed an improvement on deconvolution performance when using phantoms and real cell images.
\end{abstract}

Keywords: computational data analysis and simulation in general sciences, computational optical sectioning microscopy, deconvolution microscopy, poisson noise, Anscombe transformation.

\section{INTRODUCTION}

The visualization of the proper three-dimensional (3D) image of a biological specimen is important in many specific problems because the cell structure and its function can be strongly correlated. This aim can be achieved with the use of the computational optical sectioning microscopy (COSM) technique. In COSM, a 3D image is formed by stacking a series of two-dimensional (2D) images that are acquired by using light microscopy. With the use of a widefield or confocal microscope it is often used in fluorescence microscopy where the data represents the fluorescence concentration [1].

However, the 3D images acquired using COSM are degraded by the microscope optics. Indeed, each slice of the 3D image is blurred, i.e., it has contributions of light from other out-of-focus planes [1, 2]. As a result, the light originated from a point in the focal plane is not exactly imaged as a point. Also, the Fourier optics theory demonstrates that there is a cut-off spatial frequency which is directly determined by the shape and size of the limiting pupil in the optical system. In this sense, some image content are lost during image acquisition [3].

Besides the blurring due to the microscope optics, there are several sources of noise that decrease the quality of the images $[1,2]$. As a result of the many problems that arise when imaging living cells (for instance, to avoid photobleaching), the images are often recorded under low-level light. In these situations, the images are quantum limited and they are corrupted by Poisson noise. Other noise sources come from the charged-couple device (CCD) camera systems that are usually used to record the images. For instance, thermal noise due to electronic devices.

Correspondence to: Murillo R.P. Homem - E-mail: murillo@ufscar.br

${ }^{1}$ Departamento de Computação de Sorocaba, Universidade Federal de São Carlos, campus de Sorocaba, Sorocaba, SP, Brazil.

2 Universidade Federal de Viçosa, campus de Rio Paranaíba, Rio Paranaíba, MG, Brazil.

${ }^{3}$ Departamento de Computação, Universidade Federal de São Carlos, São Carlos, SP, Brazil. 
Since the formation of an image changes the recorded information content with respect to that of the original object, there is interest in processing images so that the results closely match the real object. The algorithms that allow a reconstruction of the distorted images and the recovery of frequencies lost in the acquisition process are called restoration or super-resolution restoration algorithms [3].

The problem of recovering the specimen function (in this case, the fluorescence concentration) from the observed blurred and noisy image is often referred to as deconvolution microscopy. Over the last 40 years, several different algorithms with different complexity and processing time have been developed to solve this problem [1]. Linear, non-iterative, true 3D deconvolution methods such as the regularized linear least squares (RLLS) algorithm [1] are the fastest, however, they cannot recover the missing Fourier components. In principle, non-linearity is necessary in order to recover the missing frequencies as was shown in reference $[1,3]$. However, if one desires a first estimate, the RLLS is able to give a good first approximation of the true image. Better approximations can be obtained with iterative and non-linear algorithms (for a comparison of several such methods see references [1] and [2]). One widely used method is the maximum likelihood expectation maximization algorithm (MLEM) applied to microscopy by several investigators [4, 5]. Algorithms based on the MLEM method provide a better combination of robustness to noise and superresolution capabilities (i.e., they can recover some of the missing frequencies) than algorithms based on different approaches.

It is important to note that most of the algorithms we have found consider an additive signal-independent noise model in its derivation. Few and more sophisticate deconvolution methods are proposed under the assumption of a Poisson noise model which is more appropriate in fluorescence microscopy. Indeed, the algorithm that is able to produce the best visual results in COSM is the MLEM which is derived under the assumption of a photon count noise. This algorithm was previously studied in many other applications where the Poisson noise is dominant. For instance, it was proposed by Richardson and Lucy (RL) $[6,7]$ for astronomical data deconvolution.

In this work we propose a pre-processing stage to reduce the Poisson noise in fluorescence optical microscopy before the restoration process using the RL algorithm. In order to accomplish that we use the non-linear Anscombe transformation and its optimum inverse [8]. The iterative RL algorithm is applied after noise minimization.

The results of the method for simulated data are compared with the ones obtained by the original algorithm. The method improved the results by obtaining higher results in improvement in signal-to-noise ratio and faster convergence than the original algorithm.

\section{IMAGE-FORMATION MODEL}

By considering the blurring as a linear, space-invariant operator and in the absence of noise, the 3D observed image $b(x, y, z)$ in fluorescence microscopy is given by [1]

$$
b(x, y, z)=f(x, y, z) * h(x, y, z),
$$

where $f(x, y, z)$ represents the fluorescence concentration of the real object (specimen) over all the spatial dimensions and $h(x, y, z)$ is the 3D incoherent point spread function (PSF) of the microscope. The symbol $*$ stands for a 3D convolution between $f(x, y, z)$ and $h(x, y, z)$. We can write equation (1) in the Fourier domain as

$$
B(u, v, w)=F(u, v, w) \cdot H(u, v, w),
$$

where $B(u, v, w), F(u, v, w)$ and $H(u, v, w)$ are the Fourier transform (FT) of the blurred observation, of the real image and of the PSF of the optical system, respectively. It can be shown that $H(u, v, w)$ - the optical transfer function (OTF), is zero valued for most of the frequencies in the Fourier domain [4]. This is due to the circular aperture of the microscope and in the region where it has non-zero values it works as a low-pass filter and in the regions where it has zero values it removes the image content in that region. In this sense, from equation (2) we can see that the observed image has lack of information that needs to be recovered.

Moreover, the repeated exposures needed to generate the 3D data will bleach the fluorophores into the specimen. The more the time to get the image, the more significant the differences between the intensities of images at the beginning and the end of the stack of $2 \mathrm{D}$ images. This effect is known as photobleaching and it is stronger when the specimen is a living cell, which does not respond well to bleach retardants. Although one can bleach correct the stacks after the acquisition, the effect of photobleaching can be minimized (not eliminated) with a short exposure time. However, the shorter the time of exposure, the lower the level of photon count we have. Under this condition, the images are subject to a kind of noise that is dependent on the signal and that can be well modeled by a Poisson distribution.

Therefore, considering a discrete version of equation (1), a voxel of the blurred and noisy observed image is given by

$$
\tilde{g}_{k}=\operatorname{Poisson}\left\{b_{k}\right\},
$$

where Pois son $\{\cdot\}$ represents a Poisson random process and $b_{k}$ is an element of the discrete version of $b(x, y, z)$. 


\section{THE ANSCOMBE TRANSFORMATION}

The proposed method for deconvolution microscopy consists of reducing the Poisson noise in the observed image before the application of the Richardson-Lucy algorithm. Since the Poisson noise is signal dependent, we propose the use of the Anscombe transformation to be able to use more standard methods for noise removal.

The Anscombe transformation (AT) transforms the signal dependent Poisson noise into an approximately Gaussian, additive, signal independent noise with zero mean and unity variance [8].

The AT on the random variable $\tilde{g}_{k}$ is given by

$$
\tilde{u}_{k}=2 \sqrt{\tilde{g}_{k}+\frac{3}{8}}=s_{k}+\tilde{n}_{k}
$$

where $\tilde{n}_{k}$ is an additive noise that is approximately independent of $s_{k}$ and $s_{k}$ is the noiseless signal on the Anscombe domain. It can be shown that $E\left[\tilde{n}_{k}\right] \simeq 0$ and $\operatorname{Var}\left[\tilde{n}_{k}\right] \simeq 1$.

From these facts, after the AT, it is possible to use well-known techniques for reducing signal independent additive noise by operating on the random variables $\tilde{u}_{k}$. For instance, we suggest using the pointwise Wiener filter that is optimum under the minimum mean square error criteria. After the noise smoothing, we return to the original variable applying the optimal inverse AT. For a detailed explanation of the optimal inverse see the reference [8].

\section{THE RICHARDSON-LUCY ALGORITHM}

The Richardson-Lucy ( $R L)$ algorithm is an iterative procedure to find the maximum-likelihood estimate $\hat{f}(x, y, z)$ of $f(x, y, z)$ given the blurred and noisy observation. It was originally proposed by Richardson [6] and Lucy [7] and also applied in COSM by several authours $[4,5]$. Later, it was demonstrated that the algorithm was able to partially recover the missing frequency components $[2,4]$.

An iteration of the $R L$ algorithm is given by

$$
\begin{aligned}
\hat{f}_{n+1}(x, y, z)= & \left\{\left[\frac{g(x, y, z)}{\hat{f}_{n}(x, y, z) * h(x, y, z)}\right] * h(x, y, z)\right\} \\
& \times \hat{f}_{n}(x, y, z)
\end{aligned}
$$

where $g(x, y, z)$ is the blurred and noisy observation.

Since the RL algorithm tends to the ill-posed maximumlikelihood solution we also consider a regularized approach based on a total-variation penalization criteria. According to this criterion, the algorithm attempts to preserve edges while smoothing the image content.
Given an initial estimate, an iteration of the total variation Richardson-Lucy (TVRL) algorithm is given by

$$
\begin{aligned}
\hat{f}_{n+1}(x, y, z)= & \left\{\left[\frac{g(x, y, z)}{\hat{f}_{n}(x, y, z) * h(x, y, z)}\right] * h(x, y, z)\right\} \\
& \times \frac{\hat{f}_{n}(x, y, z)}{1-\lambda \operatorname{div}\left(\frac{\nabla \hat{f}_{n}(x, y, z)}{\nabla \hat{f}_{n}(x, y, z)}\right)}
\end{aligned}
$$

where div is the divergence operator and $\lambda$ a constant.

\section{RESULTS AND DISCUSSIONS}

The proposed algorithm consists of reducing the Poisson noise in the observed image before the application of the RichardsonLucy procedure. To evaluate the efficiency of the method a series of experiments were carried out using Monte-Carlo simulations. A 3D phantom image was generated which represents the actual optical density of a real microscopic structure. In order to simulate a standard non-confocal microscope the phantom image was convolved with a theoretical PSF. The PSF was calculated with the Gibson and Lanni model [9] and it corresponds to a 60X oil-immersion objective lens with a numerical aperture of 1.4. We consider that the phantom has a fluorescence wavelength of $535 \mathrm{~nm}$ and the pixel size in the $x$ and $y$ directions is 0.094 microns and in the $z$ direction it is 0.25 microns. Using the blurred image, 50 realizations of a Poisson random process were conducted, and a set of blurred and noisy images were generated.

In all experiments, to avoid boundary artifacts all images were extended and padded by a reflected version of them [10].

Four cases were considered in the simulations. First, the RL and the TVRL algorithms were applied on the blurred and noisy images without any preprocessing step to reduce the Poisson noise. Later, the RL and the TVRL algorithms were applied considering the procedure based on the AT for noise reduction. For each restored image a performance measure was computed to quantify the restoration results. It is based on the improvement in signalto-noise ratio (ISNR) which is defined by the equation (7).

$$
I S N R=10 \cdot \log _{10} \frac{\|f(x, y, z)-g(x, y, z)\|^{2}}{\|f(x, y, z)-\hat{f}(x, y, z)\|^{2}}
$$

Table 1 presents the ISNR values and the iterations numbers for all the considered algorithms. They correspond to the mean values of all deconvolution results from the image set used in the simulation. Figure 1 and Figure 2 show typical visual results for a phantom and for a real cell image restoration, respectively. 
Table 1 - ISNR for the 3D reconstructed images.

\begin{tabular}{|c|c|c|c|c|}
\hline Algorithms & $\mathrm{RL}$ & TVRL & AT+RL & AT+TVRL \\
\hline ISNR (dB) & 8.1582 & 8.6035 & 8.7384 & 8.9261 \\
Iterations & 175 & 129 & 124 & 97 \\
\hline
\end{tabular}

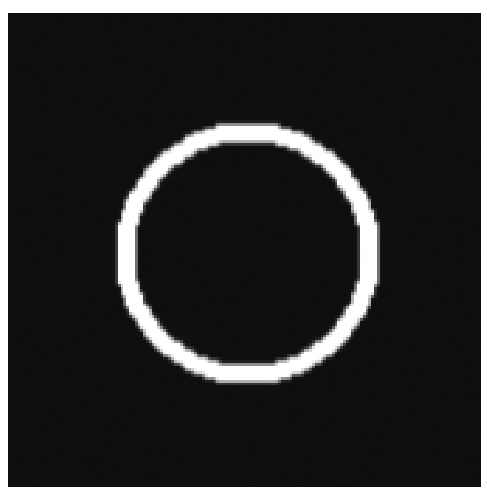

(a)

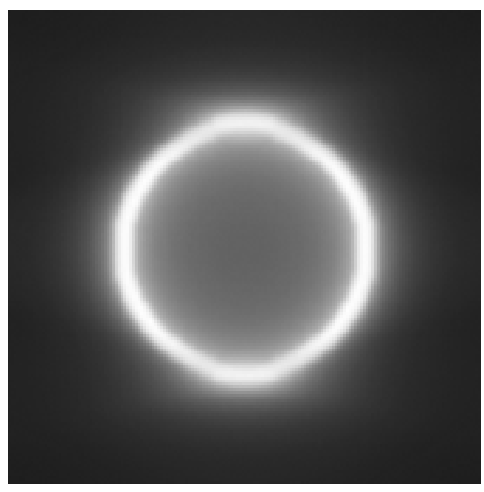

(b)

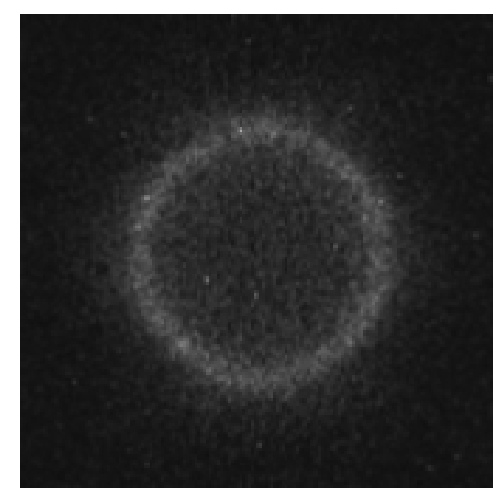

(c)

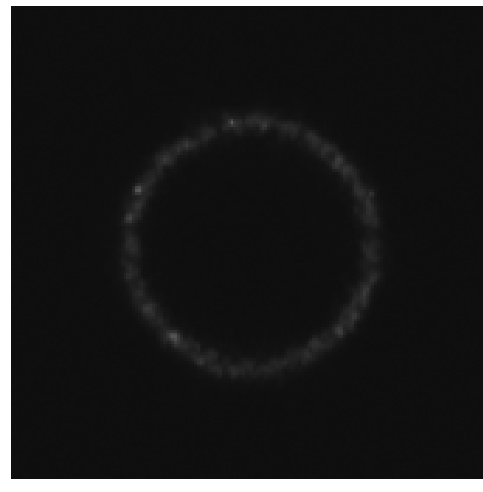

(d)

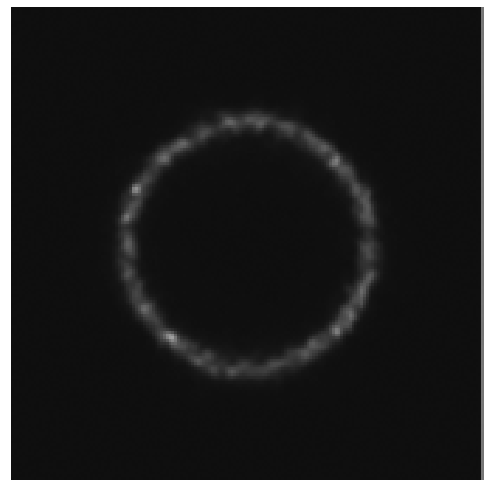

(e)

Figure 1 - (a) A section of a 3D phantom image; (b) blurred section in the axial direction (z-axis); (c) blurred and noisy section; (d) a section of the 3D restored image using the TVRL algorithm; (e) a section of the 3D restored image using the AT+TVRL algorithm.

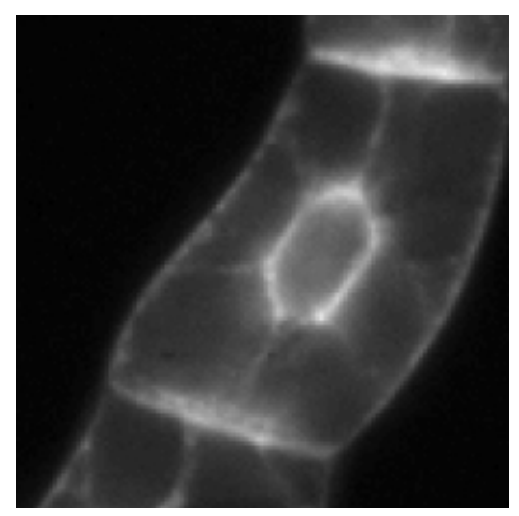

(a)

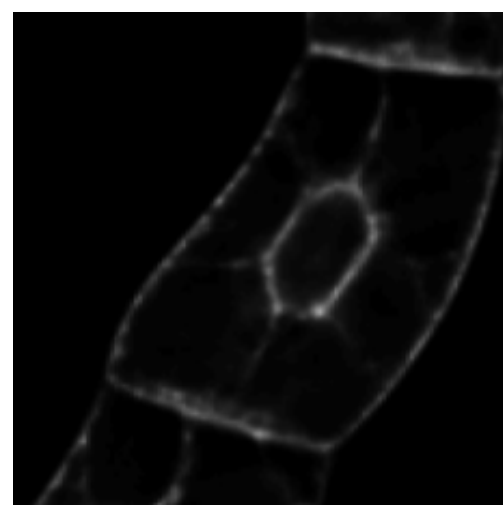

(b)

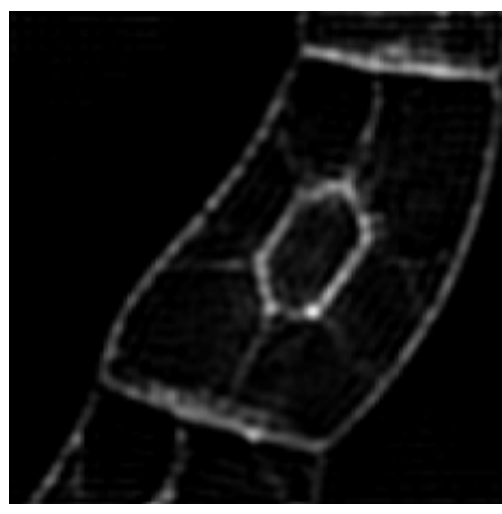

(c)

Figure 2 - (a) A real cell image; (b) restored image using the TVRL algorithm; (c) restored image using the AT+TVRL algorithm. 
The stopping criteria in all simulations was the peak value of the ISNR. From the results we can see that the noise reduction procedure as a preprocessing stage before applying the $\mathrm{RL}$ (or TVRL) algorithm is able to improve the deconvolution process following the ISNR criterion and the amount of contrast in the images. Moreover, best results are achieved with smaller iteration numbers. One can observe that distinct results can be obtained for different initial images. We have used the blurred and noisy image as the initial estimate in all cases.

The main conclusion of this work is that results provided by MLEM based algorithms derived under a Poisson noise model can be improved by a noise reduction procedure. It is important to note that after the optimal Anscombe inverse transformation we have no information about the statistics of the residual noise. However, the RL algorithm shows great robustness and also improves the results.

\section{REFERENCES}

[1] SARDER P \& NEHORAI A. 2006. Deconvolution methods for 3-D fluorescence microscopy images. IEEE Signal Processing Magazine: $32-45$.

[2] VERVEER PJ, GEMKOW MJ \& JOVIN TM. 1999. A comparison of image restoration approaches applied to three-dimensional confocal and wide-field fluorescence microscopy. J. Microsc., 193: 50-61.
[3] SEMENTILLI P, HUNT B \& NADAR M. 1993. Analysis of the limit to super-resolution in incoherent imaging. J. Opt. Soc. Am. A, 10: 2265-2276.

[4] CONCHELLO J-A. 1998. Superresolution and convergence properties of the expectation-maximization algorithm for maximumlikelihood deconvolution of incoherent images. J. Opt. Soc. Am. A, 15(10): 2609-2619.

[5] HOLMES TJ \& LIU YH. 1988. Maximum-likelihood image restoration adapted for noncoherent optical imaging. J. Opt. Soc. Am. A, 5(5): 666-673.

[6] RICHARDSON WH. 1972. Bayesian-based iterative method of image restoration. J. Opt. Soc. Am., 62(1): 55-59.

[7] LUCY LB. 1974. An iterative technique for the rectification of observed distributions. The Astronomical Journal, 79(6): 745-765.

[8] MÄKITALO M \& FOI A. 2011. Optimal inversion of the Anscombe transformation in low-count Poisson image denoising. IEEE Transactions on Image Processing, 20(1): 99-109.

[9] GIBSON FS \& LANNI F. 1991. Experimental test of an analytical model of aberration in an oil-immersion objective lens used in three-dimensional light microscopy. J. Opt. Soc. Am. A, 8(11): 1601-1613.

[10] AGHDASI F \& WARD RK. 1996. Reduction of boundary artifacts in image restoration. IEEE Transactions on Image Processing, 5(4): 611-618. 